\title{
GESTIÓN EDUCATIVA Y LIDERAZGO TRANSFORMACIONAL DE LOS DIRECTIVOS EN LA EDUCACIÓN BÁSICA REGULAR
}

\author{
EDUCATIONAL MANAGEMENT AND TRANSFORMATIONAL LEA- \\ DERSHIP OF THE MANAGERS IN REGULAR BASIC EDUCATION
}

\author{
Nely Mejía Campó ${ }^{1}$ \\ 1.* Investigadora independiente, Cajamarca, Peru. \\ Email: mejianely450@gmail.com (iD https://orcid.org/0000-0001-6328-8062
}

Como Citar: Mejía Campó, N. (2021). Gestión educativa y liderazgo transformacional de los directivos en la educación básica regular. Revista Publicando, 8(29), 79-86. https://doi.org/10.51528/rp.vol8.id2191

\begin{abstract}
RESUMEN:
La presente investigación es de nivel correlacional, tipo aplicada, de diseño no experimental, de corte transversal, de un enfoque cuantitativo y se usó el método hipotético-deductivo. Se trabajó con una muestra de 33 docentes. El objetivo principal fue determinar la relación entre la gestión educativa y el estilo de liderazgo transformacional de los directivos en la Institución Educativa N. 2076. Se presenta la hipótesis general, que permitió establecer que entre las variables gestión educativa y liderazgo transformacional, se manifiesta una alta correlación positiva de Pearson $=.794$. En la primera hipótesis específica, las variables gestión pedagógica y liderazgo transformacional establecieron una correlación alta de Pearson = .681. En cuanto a la segunda hipótesis se determinó una alta correlación positiva de Pearson= .697 entre la gestión administrativa y liderazgo transformacional. Así mismo en la tercera hipótesis específica, entre las variables gestión comunitaria y liderazgo transformacional se determinó una alta correlación positiva de Pearson $=.811$.
\end{abstract}

\section{PALABRAS CLAVE:}

Gestión educativa, Liderazgo transformacional, educación básica.

\section{ABSRTACT:}

The present research is of correlational level, applied type, non-experimental design, cross-sectional, quantitative approach and the hypothetical-deductive method was used. We worked with a sample of 33 teachers to determine the relationship between educational management and the transformational leadership style of the directors in Educational Institution N. ㅇ 2076. The general hypothesis is presented, which allowed establishing that between the variable's educational management and transformational leadership, there is a high positive correlation of Pearson $=.794$. In the first specific hypothesis, the variables pedagogical management and transformational leadership showed a high correlation of Pearson $=$ .681. Regarding the second hypothesis, a high positive correlation of Pearson $=.697$ was determined between administrative management and transformational leadership. In the third specific hypothesis, the variables community management and transformational leadership had a high positive correlation of Pearson $=.811$.

\section{KEYWORDS:}

Educational management, transformational leadership, basic education. 


\section{INTRODUCCIÓN}

$\mathrm{L}$

a gestión institucional es una actividad permanente y necesaria, utilizada en todo tipo de organización, ya sea en el sector público o privado; de ello dependerá el cumplimiento de los objetivos y finalidades propuestos en la visión y misión de dicha Institución Educativa. Este tema lo consideramos de vital importancia dentro de la actividad educativa, porque el gestor de una Institución Educativa Pública o Privada, planifica, coordina, ejecuta, controla y evalúa las necesidades y requerimientos de todas las actividades que se relacionan en la enseñanza y aprendizaje entre los profesores, estudiantes y la comunidad educativa.

Nuestra investigación parte de una premisa ¿Qué diferencia existe entre gestión y administración educativa? Porque pedir sesiones de aprendizajes, fichas de aplicación, organizadores gráficos, registros de notas y otros, no es el sentido en la expresión de gestión educativa, por eso nuestro problema busca determinar el grado de relación que existe entre la gestión y el liderazgo transformacional del directivo de nuestro centro laboral, la cual se constata en nuestras labores diarias y que no siempre, se ha desarrollado de manera tan apropiada, como para producir resultados óptimos en la labor docente y que deben repercutir en el rendimiento académico de los estudiantes.

Desde la perspectiva de un gerente educacional, se reconoce la necesidad de una gestión con base en la ejecución de planes de desarrollo institucional, en donde las actividades de planificación se tomen en consideración como eje principal frente al desarrollo educativo y se organice las metas a futuro, considerando la participación de los agentes educativos, en donde el directivo desarrolle su desempeño directriz en óptimas condiciones, sobresaliendo para ello el liderazgo transformacional, para que fortalezca y ayude a planificar el trabajo directivo en el proceso educativo aplicando una reingeniería educativa y social de la escuela y que aunando esfuerzos con la comunidad educativa encuentren el derrotero del cambio en la enseñanza y el aprendizaje de los docentes y estudiantes.

Koontz-Weihrich (1998) definen la gestión como una interacción de decisiones sobre los recursos disponibles a lo interno de la organización con sus objetivos globales y las características del entorno en que se desempeñan, esta concepción teórica permite identificar la educación y su gestión como sistemas que se integran con una serie de elementos o factores, cuyas funciones y relaciones entre sí buscan el logro de propósitos específicos y definidos.

En esa misma dirección Navarro (2012) al conceptualizar la gestión, menciona que esta tiene que ver con el desarrollo de variados ámbitos que remiten a prácticas administrativas, organizacionales, culturales, políticas, sociales, académicas y pedagógicas, las cuales son realizadas por sujetos que la conforman y que orientan la cultura de los escolares, dándose así una transformación en la institución y sus resultados.

Para nuestro interés, la calidad, en términos de servicios, es más difícil su percepción, ya que se trata de relaciones, comunicaciones y procesos y estas forman parte de la calidad de las transformaciones sociales, por lo tanto, vinculadas a valores, prejuicios, estereotipos, actitudes, motivaciones y comportamientos. Ahora bien, si nos introducimos al mejoramiento de la calidad de la educación, tenemos necesariamente que considerar que en este trabajo contamos con los sujetos (educandos, educadores y comunidad), elementos (objetivos, contenidos, métodos, medios, materiales, infraestructura, tiempo) y procesos (planeamiento, investigación, programación, implementación, ejecución y evaluación) de calidad a este conjunto llamamos calidad total.

El éxito de la calidad es responsabilidad de la gerencia, esta debe preocuparse de que la calidad total se internalice en la cultura de la organización y en crear constancia de propósitos para el mejoramiento del producto y del servicio. Estudiantes y docentes alcanzan su máximo potencial a través del mejoramiento continuo de sus labores.

Sobre la calidad educativa, Harf y Azzerboni (2006) afirman que:

Es aquella capaz de o que tiende a formar sujetos autónomos, libres dotados de las herramientas, de los conocimientos necesarios para pensar y construir una sociedad más justa, democrática e igualitaria, a partir de la consideración de sus aspectos sociales, políticos, culturales y económicos.

Dentro de este concepto, el cambio es la aceptación de que todo está interconectado en las organizaciones, porque el factor humano es el verdadero nexo de unión. De aquí nace la inclusión generalizada de la acepción "total" al considerar que todas las partes, todas las actividades, todos los procesos contribuyen a la calidad y no solo lo que aparentemente y de forma directa produce algo.

Sin embargo, el cambio esencial que se produce es considerar que el foco de toda actividad académica es el estudiante y por lo tanto, su satisfacción es lo esencial de la calidad, por lo que la mirada no se pone ahora en lo tangible, lo material, los productos sino en lo intangible que se añade al producto.

El liderazgo transformacional según Bass, Avolio y Goodheim (1987) es un proceso comportamental, comprendido por tres factores: carisma, estimulación intelectual y consi- 
deración individualizada de las necesidades de los seguidores, siendo la estimulación intelectual, la que se correlaciona significantemente con la satisfacción laboral. Los líderes transformacionales se caracterizan por motivar e inspirar en sus seguidores que están a su alrededor entusiasmo y tenacidad proporcionando significado y reto al trabajo de los seguidores. El espíritu de equipo crece. Se muestra entusiasmo y optimismo. Los líderes consiguen que los seguidores se involucren en la visión de estados futuros atractivos; ellos crean expectativas claramente comunicadas, que los seguidores desean lograr, así como demostrar compromiso con las metas y la visión compartida.

Chiavenato (2004) afirma que el liderazgo, es considerado como un fenómeno que ocurre exclusivamente en la interacción social; debe ser analizado en función de las relaciones que existen entre las personas, en una determinada estructura social, y no por el examen de una serie de características individuales. Estudios psicológicos sobre el liderazgo sostienen que buscamos en nuestros líderes la seguridad que nos proporcionaba el símbolo paterno. Y así, como conceptualizamos a nuestro padre como un ser perfecto e infalible, reproducimos esta fijación hacia nuestros líderes, considerándolos, por lo tanto, más grandes, más inteligentes y más capaces que nosotros, este fenómeno de interacción social no se debe estudiar de forma individual, sino a través de las relaciones que surgen en un determinado grupo de personas.

Liderazgo y posición formal ocupada en la organización no son sinónimos, esto quiere decir que normalmente se tiende a igualar el liderazgo con ocupar determinadas posiciones formales en la organización (gestión), soslayando el hecho de que la organización se mueve no por la posición ocupada, sino por la capacidad para implicar a otros en la cultura escolar, a esto se denomina liderazgo en la gestión educativa.

En cambio, el liderazgo transformacional establece metas y objetivos en el intento de hacer a su seguidor líder. Este proceso de transformar a los seguidores no consiste simplemente en darles la responsabilidad de llegar a una meta, se trata también de un proceso a través del cual desarrollan la capacidad de determinar su propia actuación. Este líder puede caracterizarse por tener una idea clara de los objetivos (visión), creación de motivación alta para conseguirlos (motivación de logros) y creación de dinámica que redefine la cultura del centro (introduce innovación).

Bass (2000) define al liderazgo transformacional como el comportamiento de ciertos directivos que tienden a convertir a sus profesores en líderes de la actividad educativa que llevan a cabo. Esto lo consiguen los docentes, a través de un diálogo alturado con los docentes, indicándoles el fin a encontrar, entregándoles los medios y materiales para que consigan más de lo que esperaban conseguir por ellos mismos: haciéndoles hincapié en la importancia que tienen los resultados obtenidos y motivándoles con que los objetivos de bien común de la escuela están por encima de los intereses personales.

\section{MATERIALES Y MÉTODOS}

Es una investigación básica con un diseño correlacional cuyo objetivo de la investigación es determinar la relación que existe entre las variables gestión educativa y liderazgo transformacional. La población estuvo conformada por 33 profesores entre hombres y mujeres de la I.E. N. ${ }^{\circ} 2076$ de Puente Piedra.

\section{INSTRUMENTOS}

Se emplearon dos instrumentos denominados: "Cuestionario sobre gestión educativa" y "Cuestionario sobre liderazgo transformacional". Dichos instrumentos fueron construidos en relación con las dimensiones y los procedimientos de operacionalización de las variables; fueron aplicados a la muestra para determinar su nivel de percepción sobre la dimensión gestión educativa y lo propio con la medición de liderazgo transformacional.

Tabla1. Instrumentos y técnicas

\begin{tabular}{lllc}
\hline TÉCNICA & INSTRUMENTO & ALCANCE & INFORMANTE \\
\hline Encuesta & $\begin{array}{l}\text { Cuestionario de } \\
\text { preguntas. }\end{array}$ & $\begin{array}{l}\text { Variable } \\
\text { Gestión } \\
\text { educativa }\end{array}$ & Docentes \\
& & Variable & \\
Encuesta & Cuestionario de & Liderazgo & \\
& preguntas. & transforma- & \\
& & cional. & \\
& &
\end{tabular}

Fuente: Elaboración propia (2018).

Ambos cuestionarios contenían preguntas a ser respondidas de acuerdo con sus percepciones y se dispusieron respuestas usando la escala de Likert, donde los encuestados debían marcar con un check o aspa sobre la elección de su preferencia. Las respuestas podían ser: Nunca (valor=1), Casi nunca (valor=2), Algunas veces (valor=3), Casi siempre (valor=4) y Siempre (valor=5). 
Tabla 2. Ficha técnica del instrumento para medir la variable Gestión educativa

\begin{tabular}{|c|c|}
\hline Nombre del instrumento & $\begin{array}{l}\text { Cuestionario sobre } \\
\text { Gestión educativa }\end{array}$ \\
\hline Autores & Nely Mejía Campó \\
\hline Institución o lugar & Institución Educativa 2076 \\
\hline Objetivo & $\begin{array}{l}\text { Recolectar datos sobre las per- } \\
\text { cepciones acerca de la gestión } \\
\text { educativa }\end{array}$ \\
\hline Población & Docentes \\
\hline Tamaño muestral & Docentes \\
\hline Nivel de confianza & $95.0 \%$ \\
\hline Margen de error & $5.0 \%$ \\
\hline Dimensiones & $\begin{array}{l}\text { Institucional, pedagógica, } \\
\text { administrativa, comunitaria }\end{array}$ \\
\hline Escala de medición & $\begin{array}{l}\text { Likert: 1. Nunca, 2. Casi nun- } \\
\text { ca, 3. A veces, 4. Casi siempre, } \\
\text { 5. Siempre }\end{array}$ \\
\hline Fecha de aplicación & $01 / 08 / 2018-15 / 08 / 2018$ \\
\hline Forma de administración & Individual \\
\hline Administrado a & Docentes \\
\hline Tiempo de administración & 15 minutos \\
\hline Observaciones & $\begin{array}{l}\text { Como norma de aplicación: } \\
\text { el usuario marcará cada ítem } \\
\text { conforme con sus percep- } \\
\text { ciones. }\end{array}$ \\
\hline
\end{tabular}

Fuente: Elaboración propia (2018).

Tabla 3. Ficha técnica del instrumento para medir la variable Liderazgo transformacional

\begin{tabular}{ll}
\hline Nombre del instrumento & $\begin{array}{l}\text { Cuestionario sobre } \\
\text { Gestión educativa }\end{array}$ \\
\hline Autores & NeLY MEJíA CAMPó \\
Institución o lugar & $\begin{array}{l}\text { Institución Educativa 2076 } \\
\text { Recolectar datos sobre las per- } \\
\text { Cepciones acerca del liderazgo } \\
\text { transformacional }\end{array}$ \\
Población & Docentes \\
Tamaño muestral & Docentes \\
Nivel de confianza & $95.0 \%$ \\
Margen de error & $5.0 \%$ \\
Dimensiones & $\begin{array}{l}\text { Influencia idealizada, } \\
\text { motivación inspiracional, } \\
\text { estimulación intelectual, con- } \\
\text { sideración individualizada }\end{array}$
\end{tabular}

\begin{tabular}{ll}
\hline Nombre del instrumento & $\begin{array}{l}\text { Cuestionario sobre } \\
\text { Gestión educativa }\end{array}$ \\
\hline Escala de medición & $\begin{array}{l}\text { Likert: 1. Nunca, 2. Casi nun- } \\
\text { ca, 3. A veces, 4. Casi siempre, } \\
\text { 5. Siempre }\end{array}$ \\
Fecha de aplicación & 01/08/2018-15/08/2018 \\
Forma de administración & Individual \\
Administrado a & Docentes \\
Tiempo de administración & $\begin{array}{l}15 \text { minutos } \\
\text { Observaciones }\end{array}$ \\
& $\begin{array}{l}\text { Como norma de aplicación: } \\
\text { cl usuario marcará cada ítem } \\
\text { conforme con sus percep- } \\
\text { ciones }\end{array}$ \\
\hline
\end{tabular}

Fuente: Elaboración propia (2018).

\section{VALIDACIÓN DE LOS INSTRUMENTOS}

Los dos cuestionarios fueron validados mediante la técnica del juicio de expertos, conformada por doctores y maestros de las Universidades Nacionales de San Marcos, quienes revisaron: la pertinencia, relevancia y claridad del contenido de ambos cuestionarios.

\section{CONFIABILIDAD DE LOS INSTRUMENTOS}

Tabla 4. Escala de interpretación del coeficiente de confiabilidad

\begin{tabular}{ll}
\hline Rango de valores & Nivel o magnitud \\
\hline 0.01 a 0.20 & Muy baja \\
0.21 a 0.40 & Baja \\
0.41 a 0.60 & Moderada \\
0.61 a 0.80 & Alta \\
0.81 a 1.00 & Muy alta \\
\hline
\end{tabular}

Fuente: Ruiz (2002).

Para el análisis correspondiente al instrumento Gestión educativa se tomó una muestra piloto de 20 encuestados.

Tabla 5. Confiabilidad del cuestionario Gestión educativa

\begin{tabular}{ll}
\hline Alfa de Cronbach & N de elementos \\
\hline 0,99 & 32 \\
\hline
\end{tabular}

Fuente: Procesamiento de datos obtenidos de la investigación (2018).

Tabla 6. Confiabilidad del cuestionario Liderazgo transformacional

\begin{tabular}{lc}
\hline Alfa de Cronbach & N de elementos \\
\hline 0,99 & 32 \\
\hline Fuente: Procesamiento de datos obtenidos de la investigación (2018).
\end{tabular}


Para el análisis correspondiente al instrumento liderazgo transformacional se tomó una muestra piloto de 33 encuestados.

Los coeficientes de confiabilidad obtenidos tanto de 0,99 para el cuestionario sobre gestión educativa y de 0,99 para el cuestionario sobre liderazgo transformacional denotan una muy alta consistencia entre los ítems que conforman cada cuestionario, lo que evidencia que las preguntas plasmadas en éste contribuyen de manera significativa a la definición de los conceptos que se desea investigar, ya que cuando el coeficiente se encuentra encima de 0.81 se puede aseverar que es un instrumento de muy alta confiabilidad.

Métodos de análisis de datos: Para el análisis de la información, procesamiento y presentación de datos, se ha utilizado los estadígrafos pertinentes y las medidas estadísticas correspondientes. Para el análisis descriptivo se elaboraron tablas que contienen los resultados finales de las variables y sus dimensiones, además se presentaron tablas de contingencia que relacionan a las dos variables con su respectivo gráfico de barras tridimensionales, se elaboraron utilizando el software estadístico SPSS, 23.

\section{RESULTADOS}

El presente estudio consistió en responder al objetivo general que se ha propuesto alcanzar: Determinar la relación existente entre la Gestión Educativa de los directivos y el Liderazgo Transformacional en la formación profesional de los directivos de la I.E. N. 2097 Puente Piedra, 2018.

En la hipótesis general en el presente estudio, se halló estadísticamente un coeficiente de correlación alta de Pearson de $=0.794$, entre las variables Gestión Educativa y el Liderazgo Transformacional en la población estudiada.

Tabla 7. Correlación entre Gestión Institucional y Liderazgo Transformacional

\begin{tabular}{llll}
\hline & & $\begin{array}{l}\text { Gestión in- } \\
\text { stitucional }\end{array}$ & $\begin{array}{l}\text { Liderazgo } \\
\text { transforma- } \\
\text { cional }\end{array}$ \\
\hline $\begin{array}{l}\text { Gestión } \\
\text { insti- } \\
\text { tucional }\end{array}$ & $\begin{array}{l}\text { Correlación } \\
\text { de Pearson }\end{array}$ & 1 &, $794^{* *}$ \\
& $\begin{array}{l}\text { Sig. (bilat- } \\
\text { eral) }\end{array}$ &, 000 \\
& N & 33 \\
$\begin{array}{l}\text { Liderazgo } \\
\text { transfor- } \\
\text { macional }\end{array}$ & $\begin{array}{l}\text { Correlación } \\
\text { de Pearson }\end{array}$ &, $794^{* *}$ & 1 \\
& $\begin{array}{l}\text { Sig. (bilat- } \\
\text { eral) }\end{array}$ &, 000 & \\
& $\mathrm{~N}$ & 33 & 33 \\
\hline
\end{tabular}

Nota: ${ }^{* *}$. La correlación es significativa en el nivel 0,01 (2 colas). Procesamientos de datos obtenidos de la investigación (2018).

En la hipótesis 1: La Gestión Pedagógica se relaciona significativamente con el estilo de Liderazgo Transformacional de los directivos de la muestra materia de estudio, pues se encontró que existe alta correlación positiva (0.681) entre la Gestión Pedagógica y el Liderazgo Transformacional.

Tabla 8. Correlación entre Gestión Pedagógica y Liderazgo Transformacional

\begin{tabular}{llll}
\hline & & $\begin{array}{l}\text { Gestión in- } \\
\text { stitucional }\end{array}$ & $\begin{array}{l}\text { Liderazgo } \\
\text { transforma- } \\
\text { cional }\end{array}$ \\
\hline $\begin{array}{l}\text { Gestión } \\
\text { insti- } \\
\text { tucional }\end{array}$ & $\begin{array}{l}\text { Correlación } \\
\text { de Pearson }\end{array}$ & 1 &, $681^{* *}$ \\
& $\begin{array}{l}\text { Sig. (bilat- } \\
\text { eral) }\end{array}$ &, 000 \\
& $\mathrm{~N}$ & 33 & 33 \\
$\begin{array}{l}\text { Liderazgo } \\
\text { transfor- } \\
\text { macional }\end{array}$ & $\begin{array}{l}\text { Correlación } \\
\text { de Pearson }\end{array}$ &, $681^{\star *}$ & 1 \\
& $\begin{array}{l}\text { Sig. (bilat- } \\
\text { eral) }\end{array}$ &, 000 & \\
& $\mathrm{~N}$ & 33 & 33 \\
\hline
\end{tabular}

Fuente: Procesamiento de datos obtenidos de la investigación (2018).

En el mismo sentido para la hipótesis 2: La gestión administrativa se relaciona significativamente con el estilo de liderazgo transformacional de los directivos en la Institución Educativa 2076 Puente Piedra, asimismo se encontró que existe alta correlación positiva (0.69) entre la gestión administrativa y el liderazgo transformacional.

Tabla 9. Correlación entre Gestión Educativa y Liderazgo Transformacional

\begin{tabular}{llll}
\hline & & $\begin{array}{l}\text { Gestión in- } \\
\text { stitucional }\end{array}$ & $\begin{array}{l}\text { Liderazgo } \\
\text { transforma- } \\
\text { cional }\end{array}$ \\
\hline $\begin{array}{l}\text { Gestión } \\
\text { insti- } \\
\text { tucional }\end{array}$ & $\begin{array}{l}\text { Correlación } \\
\text { de Pearson }\end{array}$ & 1 &, $697^{\star *}$ \\
& $\begin{array}{l}\text { Sig. (bilat- } \\
\text { eral) }\end{array}$ &, 000 \\
& N & \\
$\begin{array}{l}\text { Liderazgo } \\
\text { transfor- } \\
\text { macional }\end{array}$ & $\begin{array}{l}\text { Correlación } \\
\text { de Pearson }\end{array}$ &, $697^{\star *}$ & 33 \\
& $\begin{array}{l}\text { Sig. (bilat- } \\
\text { eral) }\end{array}$ &, 000 & \\
& $\mathrm{~N}$ & 33 & 33 \\
\hline
\end{tabular}

Fuente: Procesamiento de datos obtenidos de la investigación (2018) 


\section{$\theta$ \\ P U B L I C A N D O \\ I S S N $13990-9304$}

Para la hipótesis 3: La Gestión Comunitaria se relaciona significativamente con el estilo de Liderazgo Transformacional de los directivos en la Institución Educativa 2076 Puente Piedra, asimismo se encontró que existe alta correlación positiva (0.81) entre la gestión comunitaria y el liderazgo transformacional.

Tabla 10. Correlación entre Gestión Comunitaria y Liderazgo Transformacional

\begin{tabular}{llll}
\hline & & $\begin{array}{l}\text { Gestión in- } \\
\text { stitucional }\end{array}$ & $\begin{array}{l}\text { Liderazgo } \\
\text { transforma- } \\
\text { cional }\end{array}$ \\
\hline $\begin{array}{l}\text { Gestión } \\
\text { insti- } \\
\text { tucional }\end{array}$ & $\begin{array}{l}\text { Correlación } \\
\text { de Pearson }\end{array}$ & 1 &, $811^{\star *}$ \\
& $\begin{array}{l}\text { Sig. (bilat- } \\
\text { eral) }\end{array}$ &, 000 \\
& $\mathrm{~N}$ & 33 & 33 \\
$\begin{array}{l}\text { Liderazgo } \\
\text { transfor- } \\
\text { macional }\end{array}$ & $\begin{array}{l}\text { Correlación } \\
\text { de Pearson }\end{array}$ &, $811^{\star *}$ & 1 \\
& $\begin{array}{l}\text { Sig. (bilat- } \\
\text { eral) }\end{array}$ &, 000 & \\
& $\mathrm{~N}$ & 33 & 33 \\
\hline
\end{tabular}

Fuente: Procesamiento de datos obtenidos de la investigación (2018)

\section{DISCUSIÓN}

Los resultados obtenidos en la investigación muestran que existe una alta relación significativa entre las variables liderazgo transformacional y gestión educativa siendo $r=$ 0.836, asimismo, en esta investigación cada dimensión de la gestión educativa; gestión institucional (0.794); gestión administrativa (0.697); gestión pedagógica (0.681) y la gestión comunitaria (0.811) se correlaciona con el liderazgo transformacional, presentando altas correlaciones significativas con la gestión educativa. Estudios de investigación consideran al liderazgo transformacional como un factor influyente y altamente relacionado con la gestión educativa, además se plantea como un camino a seguir para la búsqueda de la excelencia. Cervera (2012) afirma que "las diversas áreas del liderazgo transformacional del director están relacionadas significativamente con las diversas áreas del Clima Organizacional en las IE del distrito de Los Olivos" (p. 105). Huillca (2015) de manera similar obtuvo una alta correlación positiva y significativa (0.842) entre las variables liderazgo transformacional y gestión pedagógica. Además, Martínez (2013) indica que "el ejercicio del liderazgo transformacional facilita al director el planteamiento de soluciones, con apoyo de sus docentes, en beneficio de la escuela y promueve el desarrollo profesional de los mismos afianzando las obligaciones profesionales de los docentes y el desarrollo de una visión conjunta institucional" (p. 2).
Por ello el liderazgo transformacional es considerado como el más adecuado para impulsar los procesos de cambio e innovación en las organizaciones, siendo una de las bases para el éxito y enfrentar retos (Almirón, Tikhomirova, Trejo y García, 2015; García, Pantoja y Duque, 2011). Los líderes transformacionales comunican una visión clara, son consideradas personas plenas, capaces de desarrollarse logrando inspirar compromiso en sus seguidores, incrementando su madurez, confianza y motivación para ir más allá de sus propios intereses (Cruz-Ortiz, Salanova y Martínez, 2013; Ganga y Navarrete, 2014; Hermosilla, Amutio, Da costa y Páez, 2016). El líder transformacional es reflexivo, proactivo, dinámico y persuasivo, genera conciencia en sus seguidores para el logro de metas colectivas y resultados notables (Contreras y Barbosa, 2013).

La investigación permite concluir que existe una correlación positiva moderada entre el liderazgo transformacional y la gestión escolar obtenida en la prueba estadística, $\mathrm{r}=0.681$. Se afirma que los líderes transformacionales fomentan relaciones cercanas con sus subordinados, caracterizadas por una pequeña distancia de poder y por la consideración individualizada de las necesidades y capacidades de sus miembros, ofreciendo desafíos y recompensas motivantes (Cervera, 2012; Morocho, 2010).

Analizando las dimensiones del liderazgo transformacional se aprecia que el $51.5 \%$ del total de los docentes opina que siempre se observa que los directivos presentan una influencia idealizada en la Institución Educativa; el 48.5\% del total de los docentes opina que casi siempre se observa motivación inspiracional por parte de los directivos en la institución educativa; el $48.5 \%$ del total de los docentes opina que casi siempre observa una estimulación intelectual por parte de los directivos; también el $48.5 \%$ del total de los docentes opina que casi siempre observa una consideración individualizada por parte de los directivos. Estos resultados se aproximan a la investigación de Martínez (2014) que refleja que los docentes participantes de su investigación identificaron 3 de 4 características de liderazgo transformacional como: influencia en el docente (91.5\%), motivación en el docente (93.5\%) y estimulación del docente (96.1\%). Además, los entrevistados reconocieron otras características como autoridad (88.9\%), poder de convencimiento $(88.9 \%)$ y apoyo en el trabajo (100\%).

Los directores para actuar con liderazgo transformacional deben ser personas con metas objetivas, con una personalidad atrayente, saber comunicar sus deseos, poseer un dinamismo ejemplar, saber actuar en diferentes contextos, según la situación que se presente ya que la vida escolar es dinámica (Martins, Cammaroto, Neris y Canelón, 2009). Por tanto, si se quiere cambiar las escuelas y con ello me- 
jorar la educación con implicancia en el enfoque ambiental, se requiere "contar con personas que ejerzan un liderazgo desde su interior; que inicien, impulsen, faciliten, gestionen y coordinen el proceso de transformación, que posean una preparación técnica adecuada y, sobre todo, con una actitud y un compromiso con la escuela, la educación y la sociedad, capaces de ponerse al frente del proceso de cambio" (Murillo, 2006, p. 2). Este pensamiento concuerda con el trabajo de investigación ya que si un director se desenvuelve bajo la ejecución de las cinco dimensiones descritas estará en la capacidad de cumplir con eficacia los seis compromisos de gestión escolar del MINEDU.

Actualmente en el Perú se habla de un liderazgo pedagógico, a los directores los denominan líderes pedagógicos; sin embargo, este término está fundamentado en los principios y características del liderazgo transformacional (MINEDU, 2014). Con ello se busca ejercer un liderazgo capaz de generar nuevos líderes en la organización, para que aporte al desarrollo de la misión y visión de la escuela. Pues la función principal del director es generar un significado del trabajo, promover y articular una visión creíble por el conjunto de personas que integren la organización (Salazar, 2006).

Finalmente, esta investigación concuerda con el planteamiento de García et al., (2011), al indicar que el liderazgo transformacional ha ganado aceptación por parte de los directivos, al ofrecerles alternativas para centrarse en comportamientos y situaciones que involucran aspectos importantes de la cultura y el clima organizacional, generando una actitud innovadora en el personal que puede contribuir a impulsar cambios estratégicos en las organizaciones.

\section{CONCLUSIONES}

- La gestión institucional se relaciona significativamente con el estilo de liderazgo transformacional de los directivos en la Institución Educativa N. ${ }^{\circ} 2076$, Puente Piedra, asimismo se aprecia que existe alta correlación positiva (0.794) entre la gestión institucional y el liderazgo transformacional.

- La gestión pedagógica se relaciona significativamente con el estilo de liderazgo transformacional de los directivos en la Institución Educativa N.o 2076, Puente Piedra, asimismo se aprecia que existe alta correlación positiva (0.68) entre la gestión pedagógica y el liderazgo transformacional.

- La gestión administrativa se relaciona significativamente con el estilo de liderazgo transformacional de los directivos en la Institución Educativa N.o 2076. Puente Piedra, asimismo se aprecia que existe alta correlación positiva (0.69) entre la gestión adminis- trativa y el liderazgo transformacional.

- La gestión comunitaria se relaciona significativamente con el estilo de liderazgo transformacional de los directivos en la Institución Educativa N. 2076. Puente Piedra, asimismo se aprecia que existe alta correlación positiva (0.81) entre la gestión comunitaria y el liderazgo transformacional.

- La gestión educativa se relaciona significativamente con el estilo de liderazgo transformacional de los directivos en la Institución Educativa N. ${ }^{\circ} 2076$. Puente Piedra, asimismo se aprecia que existe alta correlación positiva (0.83) entre la gestión educativa y el liderazgo transformacional. 


\section{삼 P U B L I C A N D O

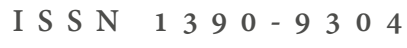

\section{REFERENCIA BIBLIOGRÁFICA}

Almirón, V., Tikhomirova, A., Trejo, A., \& García, J. (2015). Liderazgo transaccional vs Liderazgo transformacional. ReiDoCrea, 4, 24-27. Recuperado a partir de http://www.ugr.es/ reidocrea/ReiDoCrea-??Vol.4-??Art.4-??Almiron.pdf

Azzerboni Delia y Harf Ruth, (2006). Conduciendo la escuela: manual de gestión directiva y evaluación institucional. Buenos Aires. Argentina. Ediciones Novedades Educativas.

Bass, B. M. (2000): "El futuro del liderazgo en las organizaciones que aprenden”. En Actas del III Congreso Internacional del ICE de la Universidad de Deusto sobre la dirección de Centros Educativos.

Bass, B., Avolio B. y Goodheim L. (1987) Biography and the assessment of transformational leadership at the world class level. Journal of Management.

Cervera, L. E. (2012). Liderazgo Transformacional del Director y su relación con el clima organizacional en las Instituciones Educativas del Distrito de Los Olivos. Universidad Nacional Mayor de San Marcos, Lima.

Chiavenato, I. (2004). Liderazgo Bogotá. Colombia. Mc Graw Hill Interamericana Editores.

Contreras, F., \& Barbosa, D. (2013). Del liderazgo transaccional al liderazgo transformacional: implicaciones para el cambio organizacional. Revista Virtual Universidad Católica del Norte, (39), 152-164.

Cruz-Ortiz, V., Salanova, M., \& Martínez, I. M. (2013). Liderazgo transformacional: investigación actual y retos futuros. Universidad and Empresa, (25), 13-32. Recuperado a partir de http://revistas.urosario.edu. co/index.php/empresa/article/viewFile/2871/2344

Ganga, F., \& Navarrete, E. (2014). Aportaciones teóricas significativas sobre el liderazgo carismático y transformacional. Revista Venezolana de Gerencia (RVG), 19(67), 456-476.

García, M., Pantoja, M., \& Duque, L. (2011). El liderazgo Transformacional en las organizaciones: Un análisis descriptivo. JEL, 12-14, 19.

Hermosilla, D., Amutio, A., Da costa, S., \& Páez, D. (2016). El Liderazgo transformacional en las organizacio- nes: variables mediadoras y consecuencias a largo plazo. Journal of Work and Organizational Psychology, 32, 135-143.

Huillca, B. (2015). Liderazgo transformacional y desempeño docente en la especialidad de ciencias histórico - sociales del Instituto Pedagógico Nacional Monterrico. Universidad Nacional Mayor de San Marcos.

Koontz, H y Weihrich, H (1998). Administración. México. Novena Edición. Mc Graw Hill Interamericana Editores.

Martínez, Y. (2013). El liderazgo transformacional en la gestión educativa de una institución educativa pública en el distrito de Santiago de Surco. Pontificia Universidad Católica del Perú.

Martínez, Y. (2014). El liderazgo transformacional en una institución educativa pública. Educación, XXIII (44), 7-28.

Martins, F., Cammaroto, A., Neris, L. M., \& Canelón, E. del C. (2009). Liderazgo Transformacional y Gestión Educativa. Revista Electrónica "Actualidades Investigativas en Educación», 9(2), 1-27.

MINEDU. (2014). Marco de buen desempeño del directivo: directivos construyendo escuela. Recuperado a partir de https://bit.ly/3uKqZCA

Morocho, L. M. (2010). Liderazgo Transformacional y Clima Organizacional de las Instituciones Educativas de La Ciudad Satélite Santa Rosa Región Callao. Universidad San Ignacio de Loyola. Recuperado de https://bit.ly/3a61t2F

Murillo, J. F. (2006). Una Dirección Escolar para el Cambio: del Liderazgo Transformacional al Liderazgo Distribuido. REICE. Revista Iberoamericana sobre Calidad, Eficacia y Cambio en Educación, 4(4e), 11-24.

Navarro, R. (2012). La Gestión Escolar: conceptualización y revisión crítica del estado de la literatura., Enero- Junio, México, UPN. Unidad Zacatecas. Revista del taller regional de investigación educativa. Año 4, núm.7, 34, 42.

Salazar, M. A. (2006). El liderazgo transformacional ¿modelo para organizaciones educativas que aprenden? UNIrevista, 1(3), 1-12. Recuperado a partir de https://bit.ly/326Ihoj 